\title{
SARS-CoV-2 vaccination for adult patients with inflammatory bowel disease: expert consensus statement by KASID
}

Yoo Jin Lee ${ }^{1 *}$, Seong-Eun Kim²*, Yong Eun Park ${ }^{3}$, Ji Young Chang ${ }^{4}$, Hyun Joo Song ${ }^{5}$, Duk Hwan Kim ${ }^{6}$, Young Joo Yang Byung Chang Kim ${ }^{8}$, Jae Gon Lee ${ }^{9}$, Hee Chan Yang ${ }^{10}$, Miyoung Choi ${ }^{11}$, Seung-Jae Myung ${ }^{12}$, The Clinical Practice Guideline Committee of the Korean Association for the Study of the Intestinal Diseases (KASID)

${ }^{1}$ Division of Gastroenterology and Hepatology, Department of Internal Medicine, Dongsan Medical Center, Keimyung University School of Medicine, Daegu; ${ }^{2}$ Division of Gastroenterology, Department of Internal Medicine, Ewha Womans University College of Medicine, Seoul; ${ }^{3}$ Division of Gastroenterology, Department of Internal Medicine, Haeundae Paik Hospital, Inje University College of Medicine, Busan; ${ }^{4}$ Department of Health Promotion Medicine, Ewha Womans University Seoul Hospital, Ewha Womans University College of Medicine, Seoul; ${ }^{5}$ Division of Gastroenterology, Department of Internal Medicine, Jeju National University Hospital, Jeju National University College of Korea, Jeju; ${ }^{6}$ Digestive Disease Center, CHA Bundang Hospital, CHA University, Seongnam; ${ }^{7}$ Division of Gastroenterology, Department of Internal Medicine, Hallym University Chuncheon Sacred Heart Hospital, Hallym University College of Medicine, Chuncheon; ${ }^{8}$ Center for Colorectal Cancer, National Cancer Center, Goyang; ${ }^{9}$ Division of Gastroenterology, Department of Internal Medicine, Hallym University Dongtan Sacred Heart Hospital, Hallym University College of Medicine, Hwaseong; ${ }^{10}$ Division of Gastroenterology and Hepatology, Department of Internal Medicine, Jeonbuk National University Hospital, Jeonbuk National University Medical School, Jeonju; ${ }^{11}$ Division of Healthcare Technology Assessment Research, National Evidence-based Healthcare Collaborating Agency, Seoul; ${ }^{12}$ Department of Gastroenterology, Asan Medical Center, University of Ulsan College of Medicine, Seoul, Korea

Coronavirus disease 2019 (COVID-19), caused by the novel coronavirus, is threatening global health worldwide with unprecedented contagiousness and severity. The best strategy to overcome COVID-19 is a vaccine. Various vaccines are currently being developed, and mass vaccination is in progress. Despite the very encouraging clinical trial results of these vaccines, there is insufficient information on the safety and efficacy of vaccines for inflammatory bowel disease (IBD) patients facing various issues. After reviewing current evidence and international guidelines, the Korean Association for the Study of Intestinal Diseases developed an expert consensus statement on COVID-19 vaccination issues for Korean IBD patients. This expert consensus statement emphasizes that severe acute respiratory syndrome coronavirus 2 vaccination be strongly recommended for IBD patients, and it is safe for IBD patients receiving immunomodulatory therapy. (Intest Res 2022;20:171-183)

Key Words: COVID-19 vaccines; SARS-CoV-2; COVID-19; Inflammatory bowel disease

Received July 12, 2021. Revised August 21, 2021

Accepted August 22, 2021.

Correspondence to Seung-Jae Myung, Department of Gastroenterology, Asan Medical Center, University of Ulsan College of Medicine, 88 Olympic-ro 43-gil, Songpa-gu, Seoul 05505, Korea. Tel: +82-2-3010-3917, Fax: +82-2476-0824, E-mail: sjmyung@amc.seoul.kr

*These authors contributed equally to this study.

This guideline is being co-published in Intestinal Research (in English) and the Korean Journal of Gastroenterology (in Korean) for the facilitated distribution.

\section{INTRODUCTION}

Coronavirus disease 2019 (COVID-19) is a disease caused by a new strain of coronavirus, severe acute respiratory syndrome coronavirus 2 (SARS-CoV-2) that began in December 2019. The virus has infected more than 180 million people worldwide, resulting in 3,985,022 deaths as of July $6,2021 .{ }^{1}$ The COVID-19 pandemic has caused morbidity and mortality in all segments of society, especially in elderly or immunocompro- 
mised individuals, or patients with underlying medical conditions.

Inflammatory bowel disease (IBD) is an immune-mediated, chronic, inflammatory diseases of the gastrointestinal tract that includes Crohn's disease and ulcerative colitis, with increasing prevalence worldwide. ${ }^{2,3}$ The prevalences of ulcerative colitis and Crohn's disease in Korea are estimated to be 76.66 and 31.59 per 100,000 people, respectively. ${ }^{4}$ Because it is an immune-mediated disease, IBD patients are often treated with immune-modulating drugs, including corticosteroids, thiopurines, methotrexate, cyclosporine, biologics (such as anti-tumor necrosis factor [anti-TNF], anti-integrin and antiinterleukin), and small molecules (such as Janus kinase inhibitors). Patients receiving immunomodulatory therapy are more susceptible to severe and opportunistic infections. ${ }^{5,6}$ About $30 \%$ of IBD patients are elderly and one-third have comorbidities such as diabetes mellitus and hypertension, which puts them at a greater risk for severe COVID-19. ${ }^{7.8}$

After the onset of the COVID-19 pandemic, there was a sense of urgency in terms of developing a SARS-CoV-2 vaccine. As a result, various kinds of vaccines have been developed, including virus vector, messenger RNA (mRNA), and antigen-based vaccines. ${ }^{9}$ Some of these vaccines are available in Korea, whereas others are expected to be introduced soon. Although longterm data are not available, several reports from countries where large numbers of people have been vaccinated have demonstrated a decline in the COVID-19 incidence, and a reduction in severity and mortality. ${ }^{10,11}$ However, there is a lack of evidence on the efficacy and safety of SARS-CoV-2 vaccines in IBD patients, which creates confusion among patients and medical staffs. Most of the phase 3 trials excluded patients with immunemediated diseases and patients using immunosuppressive drugs. In Korea, SARS-CoV-2 vaccination for medical staffs began on February 26, 2021 and nationwide vaccination has been progressing smoothly. As the number of IBD patients receiving the vaccine is gradually increasing, these patients are making inquiries about their concerns related to the vaccine. Therefore, the clinical practice guideline committee of the Korean Association for the Study of Intestinal Diseases (KASID) reviewed the current research and international guidelines to develop an expert consensus statement on important issues related to COVID-19 vaccination for IBD patients in Korea.

The modified Delphi method was used to develop a consensus statement. ${ }^{12,13}$ A 9-point Likert scale was included in a self-administered questionnaire to obtain the agreement of 40 IBD clinical experts on different statements over e-mail (range
$1-9 ; 1=$ do not agree at all, $9=$ agree completely). Supporting data in the form of a literature review were provided with the questionnaire to obtain agreement among the experts. Statements with coefficient of variation less than 0.5 were adopted as recommendations. The statements that do not reach consensus were planned to be amended by the development committee and receive a second response from experts. However, a consensus was reached for all 11 statements in the first round and there was no need for a second round of Delphi process. However, even if it was pointed out by a few experts, the statement was partially revised with the consent of the experts for a reasonable opinion (Table 1).

Because there are limited relevant data, some recommendations were based on expert opinions, but an attempt was made to maintain a balance between the domestic context and recommendations from international guidelines. These recommendations should be applied on a case-to-case basis, depending on the individual circumstances of IBD patients. As new evidence emerges, these recommendations may be revised in the future.

\section{GENERAL ISSUES AND SAFETY}

\section{Is SARS-CoV-2 Vaccination Recommended for IBD Patients?}

Statement 1

SARS-CoV-2 vaccination is strongly recommended for IBD patients.

SARS-CoV-2 vaccines prevent COVID-19 infection by boosting the body's immune defenses. This vaccination is very important to prevent severe COVID-19. According to recent clinical reports, vaccination was effective for preventing asymptomatic as well as symptomatic infection. ${ }^{14,15}$ It is expected that the COVID-19 outbreak can be suppressed by achieving herd immunity (also called population immunity) through vaccination of the vast majority of the population. Vaccination is, therefore, very important to stop the spread of COVID-19. ${ }^{16}$

Because IBD patients were not enrolled in large-scale clinical studies of SARS-CoV-2 vaccines, there is insufficient evidence on the safety and efficacy of the vaccines in IBD patients. However, in early clinical studies of the SARS-CoV-2 vaccine, there was no evidence of an increase in the incidence of adverse reactions in patients with underlying diseases. Patients with chronic underlying diseases should be vaccinated because they are more likely to develop severe COVID-19 infection. The Korea 
Table 1. Consensus Statements on SARS-CoV-2 Vaccination for Adult IBD Patients by KASID

\begin{tabular}{|c|c|c|c|}
\hline Accepted statements & $\begin{array}{l}\text { Strength of } \\
\text { agreement (mean) }\end{array}$ & SD & CV \\
\hline 1. SARS-CoV-2 vaccination is strongly recommended for IBD patients. & 8.5 & 0.60 & 0.07 \\
\hline $\begin{array}{l}\text { 4. IBD patients being treated with biologic agents can be vaccinated against SARS-COV-2 irrespective } \\
\text { of the timing of drug administration. }\end{array}$ & 7.7 & 0.76 & 0.10 \\
\hline $\begin{array}{l}\text { 5. The efficacy of SARS-CoV-2 vaccination in IBD patients may vary depending on the therapeutic } \\
\text { agent used. }\end{array}$ & 8.3 & 0.67 & 0.08 \\
\hline $\begin{array}{l}\text { 6. In patients with IBD on high-dose systemic corticosteroid therapy, the efficacy of a SARS-CoV-2 } \\
\text { vaccine may be reduced. Therefore, consultation with medical staff is needed for the appropriate } \\
\text { timing of vaccination. }\end{array}$ & 8.2 & 0.78 & 0.10 \\
\hline $\begin{array}{l}\text { 8. Even after SARS-CoV-2 vaccination, personal hygiene practices and public health } \\
\text { recommendations should be followed strictly to prevent infection. }\end{array}$ & 8.9 & 0.43 & 0.05 \\
\hline $\begin{array}{l}\text { 9. SARS-CoV-2 vaccination in pregnant IBD patients is recommended, similar as for pregnant women } \\
\text { in the general population, in accordance with the government vaccination policy. }\end{array}$ & 7.9 & 0.71 & 0.09 \\
\hline $\begin{array}{l}\text { 10. In lactating IBD patients, SARS-CoV-2 vaccination is recommended, as with lactating women in } \\
\text { the general population, in accordance with the government vaccination policy. }\end{array}$ & 7.9 & 0.71 & 0.09 \\
\hline $\begin{array}{l}\text { 11. It is recommended that IBD patients who are planning to become pregnant receive the SARS- } \\
\text { CoV-2 vaccine prior to pregnancy. However, do not delay pregnancy for vaccination. }\end{array}$ & 7.8 & 0.84 & 0.11 \\
\hline
\end{tabular}

The response scale is a 9-Likert scale, ranging from 1 point (strongly disagree) to 9 points (strongly agree), and the closer to 9, the higher strength of agreement.

SARS-CoV-2, severe acute respiratory syndrome coronavirus 2; IBD, inflammatory bowel disease; KASID, the Korean Association for the Study of Intestinal Diseases; COVID-19, coronavirus disease 2019; SD, standard deviation; CV, coefficient of variation (SD/mean)

Disease Control and Prevention Agency (KDCA) recommend vaccination against SARS-CoV-2 for patients with chronic underlying diseases. As of June 2021, no live COVID-19 vaccine is being used in Korea. Therefore, even immunocompromised patients should be vaccinated against SARS-CoV-2 if they are eligible for the vaccine. ${ }^{9}$

Considering the previous recommendations for vaccination against viral diseases, the current non-live vaccines against SARS-CoV-2 should not be contraindicated for most IBD patients. Similar to recommendations for the general population, SARS-CoV-2 vaccination is strongly recommended for IBD patients. The International Organization for the Study of Inflammatory Bowel Disease (IOIBD) recommends that all IBD patients should be vaccinated against SARS-CoV-2, except with live or replication competent vector vaccines that may be approved in the future. ${ }^{17}$ The British Society of Gastroenterology (BSG) also recommends early vaccination for IBD patients, as the benefits of vaccination outweigh the risks, despite the reports of vaccine side effects in the general population. ${ }^{16}$

\section{Statement 2}

There is no evidence that the currently used non-live vaccines increase the risk of SARS-CoV-2 infection or IBD aggravation. The same level of caution is required for vaccine side effects in these patients as in the general population.

The SARS-CoV-2 vaccines currently being administered in Korea are either viral vector vaccines (AstraZeneca/University of Oxford and J\&J/Janssen) or mRNA vaccines (NIH-Moderna and Pfizer/BioNTech). However, all the available vaccines are non-live (Table 2).

SARS-CoV-2 vaccination in IBD patients has not been thoroughly investigated, but there has been no evidence that the vaccine increases the risk of SARS-CoV-2 infection or affects 
Table 2. SARS-CoV-2 Vaccines Currently Available in Korea as of Date of July 10, 2021

\begin{tabular}{|c|c|c|c|c|}
\hline \multirow{2}{*}{$\begin{array}{l}\text { Type of vaccine } \\
\text { Manufacturing company }\end{array}$} & \multicolumn{2}{|c|}{ mRNA vaccine } & \multicolumn{2}{|c|}{ Virus vector vaccine } \\
\hline & Pfizer-BioNTech & Moderna & AstraZeneca & Janssen Johnson \& Johnson \\
\hline Name & Comirnaty & - & $\begin{array}{l}\text { COVID-19 vaccine } \\
\text { AstraZeneca }\end{array}$ & Janssen COVID-19 vaccine \\
\hline Authorized age groups & $\geq 16$ years of age & $\geq 18$ years of age & $\geq 18$ years of age & $\geq 18$ years of age \\
\hline Doses per vial & Multiple doses (6) per vial & Multiple doses (10) per vial & Multiple doses (10) per vial & Multiple doses (5) per vial \\
\hline Schedule & 2 Doses (3 weeks apart) & 2 Doses (4 weeks apart) & 2 Doses (8-12 weeks apart) & 1 Dose \\
\hline Dosing & $0.3 \mathrm{~mL}$ IM (diluted) & $0.5 \mathrm{~mL} \mathrm{IM}$ & $0.5 \mathrm{~mL} \mathrm{IM}$ & $0.5 \mathrm{~mL} \mathrm{IM}$ \\
\hline Storage & $-90^{\circ} \mathrm{C}$ to $-60^{\circ} \mathrm{C}$ (6 months) & $-25^{\circ} \mathrm{C}$ to $-15^{\circ} \mathrm{C}$ (7 months) & $2^{\circ} \mathrm{C}$ to $8^{\circ} \mathrm{C}$ (6 months) & $-25^{\circ} \mathrm{C}$ to $-15^{\circ} \mathrm{C}$ (24 months) \\
\hline Transport & $\begin{array}{l}-90^{\circ} \mathrm{C} \text { to }-60^{\circ} \mathrm{C}(6 \text { months }) / \\
\left.2^{\circ} \mathrm{C} \text { to } 8^{\circ} \mathrm{C} \text { ( } 5 \text { days }\right)\end{array}$ & $2^{\circ} \mathrm{C}$ to $8^{\circ} \mathrm{C}$ (30 days) & $2^{\circ} \mathrm{C}$ to $8^{\circ} \mathrm{C}$ (6 months) & $2^{\circ} \mathrm{C}$ to $8^{\circ} \mathrm{C}$ (3 months) \\
\hline After first use & $2^{\circ} \mathrm{C}$ to $25^{\circ} \mathrm{C}, 6$ hours (diluted) & Room temperature, 6 hours & $\begin{array}{l}\text { Room temperature } \\
\text { (under } 30^{\circ} \mathrm{C} \text { ), } 6 \text { hours }\end{array}$ & $\begin{array}{l}2^{\circ} \mathrm{C} \text { to } 8^{\circ} \mathrm{C}, 6 \text { hours/room temper- } \\
\text { ature (under } 25^{\circ} \mathrm{C} \text { ), } 3 \text { hours }\end{array}$ \\
\hline
\end{tabular}

SARS-CoV-2, severe acute respiratory syndrome coronavirus 2; mRNA, messenger RNA; COVID-19, coronavirus disease 2019; IM, intramuscular. Korea Disease Control and Prevention Agency (https://ncv.kdca.go.kr/menu.es?mid =a12207000000). ${ }^{9}$

the immune system and leads to disease activation. Additionally, there have been no reports of severe gastrointestinal complications after SARS-CoV-2 vaccination in IBD patients. ${ }^{16}$

A study investigating the influence of the influenza vaccine on IBD patients receiving immunomodulators or biologics found a slight, self-limiting exacerbation of the disease in $3.9 \%$ of the patients following vaccination. ${ }^{18} \mathrm{~A}$ study of 80 IBD patients for several years demonstrated no long-term side effects of influenza vaccines on the disease activity of IBD. ${ }^{19}$ A study investigating the efficacy of 23-valent pneumococcal vaccination in 96 patients with IBD found no serious complications or disease exacerbations. ${ }^{20}$ Based on the previous studies, the safety of SARS-CoV-2 vaccine is assumed to be similar to that of other non-live vaccines.

Influenza, hepatitis A and B, and pneumococcal vaccines are currently recommended in IBD patients, being recognized for their safety. These vaccines also have been approved without clinical trials for IBD patients. ${ }^{16}$ Vaccine efficacy and safety issues are often reported in clinical settings where COVID-19 vaccination is already underway. However, there have been no reports of vaccine adverse events occurring more frequently in patients with IBD than in the general population.

Common adverse effects of SARS-CoV-2 vaccination include injection site reactions, such as injection site pain and redness, fever, headache, fatigue, and rash, which occur within 1-2 days following vaccination and disappear within a few days. ${ }^{21}$ Anaphylaxis was reported to occur in 4.7 cases per million population (47 of 9.94 million doses) following Pfizer vaccination and 2.5 cases per million population ( 19 of 9.94 million doses) fol- lowing Moderna vaccination in the United States. ${ }^{21}$ Thrombosis with thrombocytopenia syndrome was reported after vaccination with AstraZeneca and Janssen products, which use a viral vector, raising concerns about the vaccine safety. ${ }^{22,23}$ The European Medicine Agency (EMA) recognized the association between thrombocytopenic thrombosis and these vaccines and named it "vaccine-induced prothrombotic immune thrombocytopenia (VIPIT)."24 VIPIT is a rare adverse reaction in which thrombocytopenia accompanies thrombosis (such as cerebral sinus venous thrombosis or splanchnic vein thrombosis) within 4-28 days following vaccination. ${ }^{24,25}$ The pathophysiology is similar to heparin-induced thrombocytopenia, but it occurs independent of heparin exposure. The developmental mechanism suggested is that the vaccination-induced antibodies activate platelets, causing thrombosis and decreasing the number of available platelets. ${ }^{23,26}$ VIPIT occurred in 14.2 cases per million population for the first dose of the AstraZeneca SARS-CoV-2 vaccine, 20.1 cases per million for those aged 18-49 years, and 10.8 cases per million for those aged $\geq 50$ years. ${ }^{27}$ For the Janssen SARS-CoV-2 vaccine, VIPIT occurs at a rate of approximately 7 cases per million females of ages 1849 years, whereas it is rare in females aged $\geq 50$ years and males of all ages. ${ }^{28}$

The EMA, U.S. Centers for Disease Control and Prevention (CDC), and U.S. Food and Drug Administration temporarily withheld viral vector vaccines when VIPIT was initially recognized but eventually determined that the benefits of vaccination outweighed the risks. The KDCA recommends the monitoring of vaccinated patients for symptoms of thrombosis fol- 
lowing vaccination and has established a surveillance system for this complication.

Because of the high risk of venous thrombosis in IBD patients during active disease, ${ }^{29}$ they should be monitored closely following SARS-CoV-2 vaccination. However, there is no evidence that they are at higher risk for vaccine-induced thrombosis in IBD patients compared to the general population. It is, therefore, advised that IBD patients need the same level of caution following vaccination as the general population, and long-term ongoing studies featuring risk-and-benefit analysis are needed.

\section{Should Vaccination Be Recommended for IBD Patients on Immune-Modifying Treatment?}

\section{Statement 3}

SARS-CoV-2 vaccination is recommended for IBD patients who are being treated with immune-modifying drugs, including biologics and small molecule inhibitors.

Patients with IBD who are being treated with immunosuppressive drugs are likely to have a higher risk of developing serious COVID-19, although studies have shown conflicting results. ${ }^{30}$ An Italian study published at the beginning of the COVID-19 pandemic reported that among 79 IBD patients with COVID-19, the prognosis was not worse in those who received anti-TNF treatment. ${ }^{31}$ According to data in Surveillance Epidemiology of Coronavirus under Research Exclusion (SECURE-IBD), an international COVID-19 database for patients with IBD, the incidence of severe COVID-19 is more common in patients receiving systemic steroids than in those receiving anti-TNF therapy. ${ }^{32}$ However, extended follow-up reporting to SECUREIBD based on the analysis of 1,439 patients from 47 countries demonstrated that thiopurine monotherapy and anti-TNFthiopurine combination groups had a 4-fold higher risk of severe COVID-19 compared to the anti-TNF monotherapy group. ${ }^{33}$ To date, there has been no evidence of an association between treatment with ustekinumab, vedolizumab, or tofacitinib and the risk of severe COVID-19, but the BSG recommends cautions against infection. ${ }^{34}$ The BSG stratified the risk of serious COVID-19 in IBD patients into categories. The highest risk group stratified according to the drug being administered includes IBD patients receiving intravenous or oral steroids $(\geq 20$ mg prednisolone or equivalent per day) and those who started combination treatment with biologics and immunomodulators or systemic steroids within the previous 6 weeks. ${ }^{34}$ The moderate risk group includes patients receiving anti-TNF monotherapy, combination treatment with biologics and immunomodulators, ustekinumab, vedolizumab, thiopurines, methotrexate, calcineurin inhibitors, tofacitinib, or prednisolone at a dose of $<20 \mathrm{mg}$ or equivalent per day. ${ }^{34}$ Thus, it is reasonable to recommend SARS-CoV-2 vaccination to prevent serious COVID-19 in IBD patients receiving immune-modifying therapy. The safety of SARS-CoV-2 vaccination in IBD patients is not known. However, based on the evidence derived from other vaccines being used for infectious diseases, the KDCA recommends that immunocompromised individuals should be vaccinated against SARS-CoV-2 using non-live vaccines. SARS$\mathrm{CoV}-2$ vaccines currently being used were not tested in IBD patients in phase 2 and 3 trials, but a good safety profile was reported in the enrolled subjects. ${ }^{35-37}$ Large cohort studies are now underway examining immunogenicity after SARS-CoV-2 vaccination in IBD patients, and based on the initial data from these studies, no unexpected safety issues were found. ${ }^{38,39} \mathrm{Al}-$ though the evidence for the safety of SARS-CoV-2 vaccine is still lacking, the benefits of non-live vaccines appear to outweigh the risks. The IOIBD also recommends that all IBD patients should be vaccinated against SARS-CoV-2 regardless of the immune status, considering the poor prognosis of COVID-19 in immunocompromised IBD patients. ${ }^{17}$ Several expert groups, ${ }^{40}$ including BSG, ${ }^{16}$ recommend SARS-CoV-2 vaccination in IBD patients being treated with immune-modifying drugs.

\section{Statement 4}

IBD patients being treated with biologic agents can be vaccinated against SARS-CoV-2 irrespective of the timing of drug administration.

The evidence for optimal timing of SARS-CoV-2 vaccination in IBD patients is limited. According to the guidelines, vaccination is recommended before the initiation of immune-modifying treatment, if possible. ${ }^{41,42}$ However, IBD medication should not be delayed for vaccination. ${ }^{43}$ The BSG recommends that immunosuppressing/immunomodulating treatment should not be withheld for vaccination, and conversely, vaccination should not be deferred due to IBD medications. ${ }^{16}$ The IOIBD also recommends the early vaccination of IBD patients regardless of the timing of biologic treatment. ${ }^{17}$ Similarly, a Brazilian IBD study group proposed that the SARS-CoV-2 vaccination can be administrated irrespective of the timing and dosage of the last biologic agent received, but vaccination should be avoided on the day of biologic agent administration to allow 
the cause of any adverse effects to be distinguished. ${ }^{44}$ Therefore, IBD patients being treated with biological agents can be vaccinated regardless of the timing of drug administration. However, if possible, administering the SARS-CoV-2 vaccine on a different day than the administration of the biological agent will help to check for side effects.

\section{EFFICACY}

\section{Is SARS-CoV-2 Vaccination Effective in Patients with IBD?}

\section{Statement 5}

The efficacy of SARS-CoV-2 vaccination in IBD patients may vary depending on the therapeutic agent used.

Previous studies investigating vaccine efficacy in various infectious diseases have established that the effect of vaccination may be attenuated in IBD patients being treated with biologic agents, immunosuppressants, or corticosteroids. ${ }^{40,42,43}$ IBD patients on anti-TNF therapy had reduced seroconversion rates compared to controls in response to influenza, ${ }^{45}$ pneumococcal pneumonia, ${ }^{20,46}$ hepatitis $\mathrm{B},{ }^{47,48}$ and hepatitis A vaccines. ${ }^{49}$ These responses were further weakened with the use of thiopurines and methotrexate, alone or in combination with anti$\mathrm{TNF}^{50}$ There are few reports on the effects of vaccination in IBD patients treated with recently approved medications, such as vedolizumab, ustekinumab, and tofacitinib. It has been reported that vedolizumab, a gut-selective monoclonal antibody that targets $\alpha 4 \beta 7$ integrin, diminished the immune response to oral vaccines, but not to parenteral vaccines. ${ }^{51}$ However, partially blunted protection is considered better than no protection, and most experts recommend vaccinations to prevent various infectious diseases in IBD patients. ${ }^{40}$

There is a lack of data about the efficacy of SARS-CoV-2 vaccination in IBD patients. Early results from a large-scale IBD cohort study in the United Kingdom (impact of biologic therapy on SARS-CoV-2 infection and immunity, CLARITY) showed that the serologic response of SARS-CoV-2 vaccination is attenuated in patients receiving infliximab, and even more so in patients receiving combination treatment with anti-TNF agents and immunomodulators. ${ }^{38}$ The International study of COVID-19 Antibody Response Under Sustained immune suppression in IBD (ICARUS-IBD), an international cohort study, has reported initial results on the serologic response of SARS-CoV-2 vaccination in $48 \mathrm{IBD}$ patients. ${ }^{39}$ These results also indicate low seroconversion rates after the first dose of SARS-CoV-2 vaccine in patients treated with immune-modifying therapy. ${ }^{38,39}$ However, in both studies, the immune response was found to be adequate in patients who had a previous SARS-CoV-2 infection or had completed 2-dose vaccination. ${ }^{38,39}$ The vaccines used in these 2 studies were mRNA and viral vector vaccines (Pfizer, Moderna, and AstraZeneca). Based on these findings, it is suggested that precautions to prevent SARS-CoV-2 infection should be strictly followed until patients receive the second vaccine dose, and the recommended immunization schedule should be followed. Even after 2-dose vaccination, a small number of patients failed to form antibodies. ${ }^{38}$ However, the role of antibody titer measurements after vaccination and booster vaccinations in patients with low titers has not yet been established. It is also unclear whether the antibody titer is a reliable indicator for evaluating immune defense against SARS$\mathrm{CoV}-2$. Further studies are needed to determine whether antibody testing is necessary in high-risk IBD patients and if any changes in the vaccination schedule are required to elicit the maximum efficiency from SARS-CoV-2 vaccination. Among the currently available SARS-CoV-2 vaccines in Korea, the Pfizer, Moderna, and AstraZeneca vaccines have to be administrated twice, whereas the Janssen vaccine has to be administered once. The KDCA advices that it is important to maintain the recommended interval between vaccinations and complete 2-dose vaccinations because the immune response is insufficient if only 1 dose of a 2-dose vaccine is administered.

\section{Statement 6}

In patients with IBD on high-dose systemic corticosteroid therapy, the efficacy of a SARS-CoV-2 vaccine may be reduced. Therefore, consultation with medical staff is needed for the appropriate timing of vaccination.

As reported in influenza vaccine $\mathrm{s}^{52}$ and hepatitis B vaccine $\mathrm{v}^{53}$ studies, the immunogenicity induced after vaccination can be significantly reduced in IBD patients on high-dose systemic corticosteroid therapy. Therefore, the BSG recommends that SARS-CoV-2 vaccines should be administered after reducing the corticosteroids used to the lowest possible dose. ${ }^{16}$ The IOIBD recommends that IBD patients on systemic corticosteroid therapy should be counseled regarding the reduced efficacy of the vaccine. ${ }^{17}$

Statement 7

Influenza and pneumococcal vaccinations are highly recommended for IBD patients in the COVID-19 pandemic era. 
IBD patients have a higher risk of respiratory infections such as influenza and pneumococcal pneumonia compared to the general population. ${ }^{40,54}$ Patients with IBD who are receiving immunomodulating treatment are particularly at risk for these infections. ${ }^{55,56}$ Therefore, influenza and pneumococcal vaccines are highly recommended for IBD patients. ${ }^{57}$ However, based on a survey conducted in Korea, the vaccination rates for influenza and pneumococcal pneumonia were $44.2 \%$ and $4.2 \%$, respectively; and $30 \%$ of IBD patients do not recognize the importance of the vaccinations. ${ }^{54}$

The U.S. Advisory Committee on Immunization Practices (ACIP) has predicted that influenza and SARS-CoV-2 will be simultaneously prevalent in the winter of $2021 .{ }^{58}$ It is difficult to differentiate these 2 conditions as they have identical symptoms, such as fever, cough, and dyspnea. Simultaneous infections with SARS-CoV-2 and other respiratory pathogens may increase the morbidity and mortality of COVID- $19 .^{40}$ Therefore, Several expert groups ${ }^{40,43,59}$ including the COVID-19 European Crohn's and Colitis Organisation (COVID-19 ECCO) $)^{60}$ and $\mathrm{BSG}^{16}$ strongly recommend influenza and pneumococcal vaccines for all unvaccinated IBD patients.

The guidelines of the KDCA recommend maintaining an interval of at least 14 days between SARS-CoV-2 and other vaccines. ${ }^{9}$ The re-administration of SARS-CoV-2 vaccine is not recommended if it is inadvertently administered at the same time as, or within 14 days of another vaccine. ${ }^{9}$ The ACIP recommends that influenza vaccination should be deferred in patients with COVID-19 until the signs of COVID-19 disappear. ${ }^{58}$

\section{Statement 8}

Even after SARS-CoV-2 vaccination, personal hygiene practices and public health recommendations should be followed strictly to prevent infection.

The preventive effect of SARS-CoV-2 vaccination is $62 \%$ to $95 \%$, and there is still insufficient information about the duration of this effect. No vaccine developed to date can completely prevent the transmission of SARS-CoV-2. If immunity does not develop fully after vaccination or decreases over time, patients can still be infected with SARS-CoV-2. ${ }^{9,40}$ Therefore, the most effective strategy to prevent COVID-19 is to maintain personal hygiene practices, such as hand-washing, wearing masks, and social distancing. ${ }^{40,59}$ IBD patients undergoing immunomodulatory therapy may have lower immunogenicity than the general population, so even after SARS-CoV-2 vaccination, these precautions should be continued.

\section{SARS-CoV-2 VACCINATION FOR PREGNANT AND} LACTATING WOMEN

\section{Is SARS-CoV-2 Vaccination Recommended for Pregnant and Lactating IBD Patients?}

\section{Statement 9}

SARS-CoV-2 vaccination in pregnant IBD patients is recommended, similar as for pregnant women in the general population, in accordance with the government vaccination policy.

Pregnant women are at a greater risk for severe morbidity due to COVID-19 compared to non-pregnant individuals. Factors and pre-existing comorbidities such as maternal age $>40$ years, obesity, hypertension, and diabetes mellitus appear to be associated with severe COVID-19 in pregnant women. ${ }^{61,62}$ Moreover, pregnant women with COVID-19 have been reported to be 3 times more likely to have poor perinatal outcomes, such as premature birth. ${ }^{61,62}$ The vertical transmission of SARS-CoV-2 has been reported in $3.2 \%$ of infected mothers in the third trimester. ${ }^{63}$ Thus, COVID-19 prevention is vital to protect both the mother and fetus.

Non-live vaccination during pregnancy has been recommended to prevent maternal and infant morbidity from infectious diseases. ${ }^{64}$ In particular, maternal immunization for influenza and pertussis has a proven role in preventing fetus and infant infections. ${ }^{65,66}$ Although the mechanism of action of SARS-CoV-2 vaccines is different from that of both influenza and pertussis vaccines, the currently available SARS-CoV-2 vaccines are also non-live vaccines. As there have been no reports of harmful effects from non-live vaccines for other infectious diseases during pregnancy, the SARS-CoV-2 vaccine is also believed to be safe for pregnant women. ${ }^{64,67}$ However, there are very limited data on SARS-CoV-2 vaccination during pregnancy because pregnant women were excluded from clinical trials of the SARS-CoV-2 vaccines.

The SARS-CoV-2 vaccines currently available in Korea were not found to interact with recipient DNA or cause genetic modifications. ${ }^{68,69}$ In addition, no significant safety issues have been observed in animal studies on SARS-CoV-2 vaccines. ${ }^{69,70}$

As of July 06, 2021, 130,435 participants who self-identified as pregnant at the time of vaccination, or shortly thereafter, have been registered with the CDC's smartphone-based tool known as v-safe to investigate the safety of SARS-CoV-2 vaccination. ${ }^{71}$ Among them, 5,103 pregnant participants were en- 
rolled in the v-safe COVID-19 vaccine-pregnancy registry after consenting in a separate round of registration. ${ }^{71}$ According to initial v-safe data, in which vaccination results for 35,691 pregnant women were tracked between December 14, 2020 and February 28, 2021, injection site pain was more common in pregnant women, but systemic adverse reactions such as headache, muscle pain and fever were less frequently reported than in non-pregnant women. ${ }^{72}$ Although these are preliminary data, the vaccinated pregnant women in this study did not appear to be at increased risk of adverse pregnancy and neonatal outcomes from indirect comparisons with data obtained before the COVID-19 pandemic. $^{72}$

A prospective cohort study that compared the immune response after mRNA vaccination in 84 pregnant, 31 lactating, and 16 non-pregnant women showed robust humoral immunity in pregnant and lactating women, with immunogenicity and reactogenicity similar to those of non-pregnant women. SARS-CoV-2 antibody titers in vaccinated pregnant and lactating women were significantly higher than those in naturally infected pregnant individuals. ${ }^{73}$ In addition, vaccine-generated antibodies were present in umbilical cord blood and breast milk, suggesting that maternal vaccination may have the potential to prevent SARS-CoV-2 infection in the fetus or newborn. $^{73}$

Because clinical evidence is still lacking, academic societies and expert groups have taken a cautious position on vaccination during pregnancy, but most of them suggest that SARS$\mathrm{CoV}-2$ vaccination is possible even in pregnant women. The World Health Organization (WHO) recommends SARS-CoV-2 vaccination in pregnant women when the benefits of vaccination outweigh the potential risks, i.e., in cases of mothers with underlying predisposing conditions or those at high risk for severe COVID-19. ${ }^{74}$ The CDC approves of SARS-CoV-2 vaccination during pregnancy but recommends that pregnant women be informed of the benefits and risks of vaccination, and that each pregnant woman should choose whether or not to vaccinate herself. ${ }^{69}$ Meanwhile, the Society of Obstetricians and Gynaecologists of Canada recommends that all pregnant women should be offered a SARS-CoV-2 vaccine at any time during pregnancy, if they are eligible and there are no contraindications. ${ }^{68}$ The Joint Committee on Vaccination and Immunisation (JCVI) initially issued a recommendation that pregnant women at high risk of SARS-CoV-2 infection should consider getting vaccinated even during pregnancy. ${ }^{75}$ The advice was updated on April 16, 2021 to recommend that SARS-CoV-2 vaccination should be offered to all pregnant women. ${ }^{76}$
Thrombosis has been reported after the administration of viral vector vaccines (AstraZeneca and Janssen) and occurs more commonly in women. ${ }^{28,77}$ Nevertheless, because these thrombotic side effects are very rare, the EMA and UK Medicines and Healthcare Products Regulatory Agency have suggested that the benefits of vaccination likely outweigh the risks. ${ }^{78}$ Pregnancy itself is a risk factor for thrombosis, but there is currently no evidence to suggest an increased risk of thrombosis after SARS-CoV-2 vaccination in pregnant women. ${ }^{77}$ Pregnant women should be aware of the possible risks associated with vaccination, and healthcare providers should monitor them closely. ${ }^{77}$

Although no clinical data on the efficacy and safety of vaccination in pregnant women with IBD are available, the IOIBD recommends that pregnant women with IBD should be vaccinated against SARS-CoV-2 in the same way as other pregnant women and in accordance with the local recommendations. ${ }^{17}$ The KDCA has excluded pregnant women from SARS-CoV-2 vaccination as of June 2021 due to the lack of clinical data on the safety and efficacy of the vaccines in pregnant women. However, there is a possibility that SARS-CoV-2 vaccination may be recommended for pregnant women as more information on vaccine safety becomes available. ${ }^{79}$ Medical staff are encouraged to provide sufficient information about the risks and benefits of SARS-CoV-2 vaccination during pregnancy prior to administering the vaccine.

\section{Statement 10}

In lactating IBD patients, SARS-CoV-2 vaccination is recommended, as with lactating women in the general population, in accordance with the government vaccination policy.

Because breastfeeding women were excluded from the initial clinical trials of SARS-CoV-2 vaccines, there is still insufficient evidence regarding the influence of vaccination on breastfeeding women and newborns. However, there are no reported risks associated with existing non-live vaccines in breastfeeding women, and SARS-CoV-2 is not expected to infect infants through breast milk. .7777 $^{-}$

Because of the numerous benefits of breastfeeding, the UK JCVI, ${ }^{75} \mathrm{CDC}^{69}$ American College of Obstetricians and Gynecologists (ACOG) and Society of Maternal-Fetal Medicine, ${ }^{80}$ European Board and College of Obstetrics and Gynecology, ${ }^{77}$ and Society of Obstetricians and Gynaecologists of Canada ${ }^{68}$ propose that it is possible to initiate or continue breastfeeding in mothers vaccinated against SARS-CoV-2. The KDCA also 
recommends that nursing mothers should be vaccinated against SARS-CoV-2 when they become eligible in the government vaccination program.

A study investigating changes in immunoglobulin (Ig) A and IgG in the breast milk of 84 lactating women at 2-6 weeks after the first vaccination found high titers of antibodies. The secretion of IgA into breast milk became evident from week 2 and that of IgG from week 4 after vaccination. There were no serious adverse reactions in both nursing mothers and infants during the study period, and antibodies in the breast milk exhibited strong neutralizing effects, suggesting the potential to protect infants from SARS-CoV-2 infection. ${ }^{81}$

Although there have been no reports on the safety and efficacy of vaccination in lactating patients with IBD, the IOIBD recommends that breastfeeding IBD patients should be vaccinated against SARS-CoV-2 in accordance with local recommendations for the general population. ${ }^{17}$ Therefore, SARS$\mathrm{CoV}-2$ vaccination in lactating IBD patients is recommended equally as with lactating women in the general population, considering the fact that SARS-CoV-2 vaccination should be strongly recommended for IBD patients, and that breastfeeding has prominent health benefits for infants.

\section{Statement 11}

It is recommended that IBD patients who are planning to become pregnant receive the SARS-CoV-2 vaccine prior to pregnancy. However, do not delay pregnancy for vaccination.

There is no evidence that SARS-CoV-2 vaccination causes infertility. ${ }^{80,82}$ The WHO, ${ }^{74} \mathrm{CDC}^{69} \mathrm{JCVI}^{75}$ and ACOG and Society of Maternal-Fetal Medicine ${ }^{80}$ state that there is no need for a pregnancy test before SARS-CoV-2 vaccination nor a need to delay pregnancy due to vaccination. Contraception is not required after vaccination, ${ }^{80}$ and the ACOG advises that if pregnancy were to be confirmed after the first vaccination in a vaccine schedule requiring 2 vaccinations, the second vaccination should proceed as planned. ${ }^{80}$

The IOIBD recommends that IBD patients who are planning to become pregnant should receive the SARS-CoV-2 vaccine before pregnancy, but the patients should not delay pregnancy for the sake of vaccination. ${ }^{17}$

\section{CONCLUSION}

There is a lack of high-quality evidence on SARS-CoV-2 vaccination in IBD patients, and there are several unresolved ques- tions, such as the duration of immunity and long-term immunogenicity of the vaccines, long-term safety, and the impacts of new variants after vaccination. However, to protect both individuals and their communities from COVID-19, SARS-CoV-2 vaccination is strongly recommended for IBD patients. Even in IBD patients receiving immunomodulatory treatment, the SARS-CoV-2 vaccine can be safely administered regardless of the injection timing of the medication, although the efficacy of the vaccine may be reduced. It is necessary to educate patients with IBD, especially those receiving immunomodulatory treatment, such that they follow the recommended immunization schedule. Personal hygiene and prevention protocols, including social distancing, should be strictly followed even after vaccination. The importance of pneumococcal and influenza vaccination in IBD patients should be emphasized to prevent coinfection with SARS-CoV-2.

Further studies on SARS-CoV-2 vaccination are needed to determine the optimal prophylactic strategies for patients with IBD. As research on SARS-CoV-2 vaccines is being actively conducted worldwide, these recommendations may be revised depending on the results of these studies. It is hoped that these recommendations will facilitate communication about SARSCoV-2 vaccination not only with IBD patients but also among healthcare providers.

\section{ADDITIONAL INFORMATION}

\section{Funding Source}

The authors received no financial support for the research, authorship, and/or publication of this article.

\section{Conflict of Interest}

Myung SJ is an editorial board member of the journal but was not involved in the peer reviewer selection, evaluation, or decision process of this article. No other potential conflicts of interest relevant to this article were reported.

\section{Author Contribution}

Conceptualization: Myung SJ. Data curation: Lee YJ, Kim SE, Park YE, Choi M. Formal analysis: Kim SE, Choi M. Methodology: Kim SE, Park YE, Chang JY, Song HJ, Kim DH, Yang YJ, Kim BC, Lee JG, Yang HC, Choi M. Project administration: Kim SE, Myung SJ. Resources: Myung SJ. Supervision: Myung SJ. Validation: Park YE, Chang JY, Song HJ, Kim DH, Yang YJ, Kim BC, Lee JG, Yang HC, Myung SJ. Visualization: Lee YJ, Park YE, Chang JY, Song HJ, Kim DH, Yang YJ, Kim BC, Lee JG, Yang HC, Choi 
M. Writing - original draft: Lee YJ, Kim SE. Writing - review \& editing: Lee YJ, Kim SE, Park YE, Chang JY, Song HJ, Kim DH, Yang YJ, Kim BC, Lee JG, Yang HC, Choi M. Approval of final manuscript: all authors.

\section{ORCID}

Lee $\mathrm{YJ}$

Kim SE

Park YE

Chang JY

Song $\mathrm{HJ}$

Kim DH

Yang YJ

Kim BC

Lee JG

Yang HC

Choi M

Myung SJ https://orcid.org/0000-0003-1799-0146 https://orcid.org/0000-0002-6310-5366 https://orcid.org/0000-0003-4274-8204 https://orcid.org/0000-0002-7951-456X https://orcid.org/0000-0002-2561-555X https://orcid.org/0000-0003-3841-5802 https://orcid.org/0000-0001-6325-1104 https://orcid.org/0000-0003-3164-5824 https://orcid.org/0000-0003-2930-8159 https://orcid.org/0000-0001-9847-5343 https://orcid.org/0000-0002-2424-9965 https://orcid.org/0000-0003-0585-4016

\section{REFERENCES}

1. World Health Organization. WHO Coronavirus (COVID-19) Dashboard [Internet]. c2021 [cited 2021 Jul 6]. https://covid19.who.int/.

2. Ng SC, Shi HY, Hamidi N, et al. Worldwide incidence and prevalence of inflammatory bowel disease in the 21st century: a systematic review of population-based studies. Lancet 2017; 390:2769-2778.

3. Yen HH, Weng MT, Tung CC, et al. Epidemiological trend in inflammatory bowel disease in Taiwan from 2001 to 2015: a nationwide populationbased study. Intest Res 2019;17:54-62.

4. Park SH, Kim YJ, Rhee KH, et al. A 30-year trend analysis in the epidemiology of inflammatory bowel disease in the Songpa-Kangdong district of Seoul, Korea in 1986-2015. J Crohns Colitis 2019;13:1410-1417.

5. Kirchgesner J, Lemaitre M, Carrat F, Zureik M, Carbonnel F, Dray-Spira R. Risk of serious and opportunistic infections associated with treatment of inflammatory bowel diseases. Gastroenterology 2018;155:337-346.

6. Lichtenstein GR, Feagan BG, Cohen RD, et al. Serious infection and mortality in patients with Crohn's disease: more than 5 years of follow-up in the TREAT registry. Am J Gastroenterol 2012;107:1409-1422.

7. D'Amico F, Danese S, Peyrin-Biroulet L. Systematic review on inflammatory bowel disease patients with coronavirus disease 2019: it is time to take stock. Clin Gastroenterol Hepatol
2020;18:2689-2700.

8. D'Amico F, Rabaud C, Peyrin-Biroulet L, Danese S. SARS-CoV-2 vaccination in IBD: more pros than cons. Nat Rev Gastroenterol Hepatol 2021;18:211-213.

9. Korea Disease Control and Prevention Agency. Q\&A for SARSCoV-2 Vaccination [Internet]. c2021 [cited 2021 Jul 10]. https:// ncv.kdca.go.kr/menu.es?mid=a12207000000.

10. Amit S, Regev-Yochay G, Afek A, Kreiss Y, Leshem E. Early rate reductions of SARS-CoV-2 infection and COVID-19 in BNT162b2 vaccine recipients. Lancet 2021;397:875-877.

11. Frenck RW Jr, Klein NP, Kitchin N, et al. Safety, immunogenicity, and efficacy of the BNT162b2 COVID-19 vaccine in adolescents. N Engl J Med 2021;385:239-250.

12. Nair R, Aggarwal R, Khanna D. Methods of formal consensus in classification/diagnostic criteria and guideline development. Semin Arthritis Rheum 2011;41:95-105.

13. Hong KS, Ko SB, Yu KH, et al. Update of the Korean clinical practice guidelines for endovascular recanalization therapy in patients with acute ischemic stroke. J Stroke 2016;18:102113.

14. Haas EJ, Angulo FJ, McLaughlin JM, et al. Impact and effectiveness of mRNA BNT162b2 vaccine against SARS-CoV-2 infections and COVID-19 cases, hospitalisations, and deaths following a nationwide vaccination campaign in Israel: an observational study using national surveillance data. Lancet 2021; 397:1819-1829

15. Hall VJ, Foulkes S, Saei A, et al. COVID-19 vaccine coverage in health-care workers in England and effectiveness of BNT162b2 mRNA vaccine against infection (SIREN): a prospective, multicentre, cohort study. Lancet 2021;397:1725-1735.

16. Alexander JL, Moran GW, Gaya DR, et al. SARS-CoV-2 vaccination for patients with inflammatory bowel disease: a British Society of Gastroenterology Inflammatory Bowel Disease section and IBD Clinical Research Group position statement. Lancet Gastroenterol Hepatol 2021;6:218-224.

17. Siegel CA, Melmed GY, McGovern DP, et al. SARS-CoV-2 vaccination for patients with inflammatory bowel diseases: recommendations from an international consensus meeting. Gut 2021;70:635-640.

18. Rahier JF, Papay P, Salleron J, et al. H1N1 vaccines in a large observational cohort of patients with inflammatory bowel disease treated with immunomodulators and biological therapy. Gut 2011;60:456-462.

19. Mamula P, Markowitz JE, Piccoli DA, Klimov A, Cohen L, Baldassano RN. Immune response to influenza vaccine in pediatric patients with inflammatory bowel disease. Clin Gastro- 
enterol Hepatol 2007;5:851-856.

20. Fiorino G, Peyrin-Biroulet L, Naccarato P, et al. Effects of immunosuppression on immune response to pneumococcal vaccine in inflammatory bowel disease: a prospective study. Inflamm Bowel Dis 2012;18:1042-1047.

21. Korea Disease Control and Prevention Agency. Q\&A for SARS-CoV-2 Vaccination [Internet]. c2021 [cited 2021 Jul 10]. https://ncv.kdca.go.kr/menu.es?mid=a12208000000.

22. See I, Su JR, Lale A, et al. US case reports of cerebral venous sinus thrombosis with thrombocytopenia after Ad26.COV2.S vaccination, March 2 to April 21, 2021. JAMA 2021;325:24482456.

23. Greinacher A, Thiele T, Warkentin TE, Weisser K, Kyrle PA, Eichinger S. Thrombotic thrombocytopenia after ChAdOx1 nCov-19 vaccination. N Engl J Med 2021;384:2092-2101.

24. European Medicines Agency. AstraZeneca's COVID-19 vaccine: EMA finds possible link to very rare cases of unusual blood clots with low blood platelets [Internet]. c2021 [cited 2021 Jun 14]. https://www.ema.europa.eu/en/news/astrazenecas-covid-19-vaccine-ema-finds-possible-link-very-rare-casesunusual-blood-clots-low-blood.

25. Expert Haematology Panel. Guidance produced from the Expert Haematology Panel (EHP) focussed on Covid-19 vaccine induced thrombosis and thrombocytopenia (VITT). c2021 [cited 2021 Jun 14]. https://b-s-h.org.uk/media/19530/guidance-version-13-on-mngmt-of-thrombosis-with-thrombocytopenia-occurring-after-c-19-vaccine_20210407.pdf.

26. Greinacher A, Thiele T, Warkentin TE, Weisser K, Kyrle P, Eichinger S. A prothrombotic thrombocytopenic disorder resembling heparin-induced thrombocytopenia following coronavirus-19 vaccination. Res Sq [Preprint]. 2021 [cited 2021 Jun 16]. https://doi.org/10.21203/rs.3.rs-362354/v1.

27. Medicines and Healthcare Products Regulatory Agency. Coronavirus vaccine-weekly summary of Yellow Card reporting [Internet]. c2021 [cited 2021 Jul 1]. https://www.gov.uk/government/publications/coronavirus-covid-19-vaccine-adversereactions/coronavirus-vaccine-summary-of-yellow-card-reporting.

28. Centers for Disease Control and Prevention. Johnson \& Johnson's Janssen COVID-19 vaccine overview and safety [Internet]. c2021 [cited 2021 Jun 23]. https://www.cdc.gov/coronavirus/ 2019-ncov/vaccines/different-vaccines/janssen.html.

29. Grainge MJ, West J, Card TR. Venous thromboembolism during active disease and remission in inflammatory bowel disease: a cohort study. Lancet 2010;375:657-663.

30. Koroku M, Omori T, Kambayashi H, et al. Granulocyte and monocyte apheresis therapy for patients with active ulcerative colitis associated with COVID-19. Intest Res 2021 Mar 12 [Epub]. https://doi.org/10.5217/ir.2020.00148.

31. Bezzio C, Saibeni S, Variola A, et al. Outcomes of COVID-19 in 79 patients with IBD in Italy: an IG-IBD study. Gut 2020;69: 1213-1217.

32. Brenner EJ, Ungaro RC, Gearry RB, et al. Corticosteroids, but not TNF antagonists, are associated with adverse COVID-19 outcomes in patients with inflammatory bowel diseases: results from an international registry. Gastroenterology 2020; 159:481-491.

33. Ungaro RC, Brenner EJ, Gearry RB, et al. Effect of IBD medications on COVID-19 outcomes: results from an international registry. Gut 2021;70:725-732.

34. Kennedy NA, Jones GR, Lamb CA, et al. British Society of Gastroenterology guidance for management of inflammatory bowel disease during the COVID-19 pandemic. Gut 2020;69:984990.

35. Polack FP, Thomas SJ, Kitchin N, et al. Safety and efficacy of the BNT162b2 mRNA COVID-19 vaccine. N Engl J Med 2020; 383:2603-2615.

36. Voysey M, Clemens SAC, Madhi SA, et al. Safety and efficacy of the ChAdOx1 nCoV-19 vaccine (AZD1222) against SARSCoV-2: an interim analysis of four randomised controlled trials in Brazil, South Africa, and the UK. Lancet 2021;397:99-111.

37. Baden LR, El Sahly HM, Essink B, et al. Efficacy and Safety of the mRNA-1273 SARS-CoV-2 Vaccine. N Engl J Med 2021;384: 403-416.

38. Kennedy NA, Lin S, Goodhand JR, et al. Infliximab is associated with attenuated immunogenicity to BNT162b2 and ChAdOx1 nCoV-19 SARS-CoV-2 vaccines in patients with IBD. Gut 2021;70:1884-1893.

39. Wong SY, Dixon R, Martinez Pazos V, et al. Serologic response to messenger RNA coronavirus disease 2019 vaccines in inflammatory bowel disease patients receiving biologic therapies. Gastroenterology 2021;161:715-718.

40. Melmed GY, Rubin DT, McGovern DPB. Winter is coming! Clinical, immunologic, and practical considerations for vaccinating patients with inflammatory bowel disease during the coronavirus disease-2019 pandemic. Gastroenterology 2021; 160:639-644.

41. Rubin LG, Levin MJ, Ljungman P, et al. 2013 IDSA clinical practice guideline for vaccination of the immunocompromised host. Clin Infect Dis 2014;58:309-318.

42. Park SK, Choi CH, Chun J, et al. Prevention and management of viral hepatitis in inflammatory bowel disease: a clinical prac- 
tice guideline by the Korean Association for the Study of Intestinal Diseases. Intest Res 2020;18:18-33.

43. Crosby S, Schuh MJ, Caldera F, Farraye FA. Vaccination of patients with inflammatory bowel disease during the COVID-19 pandemic. Gastroenterol Hepatol (N Y) 2021;17:18-30.

44. Queiroz N, Teixeira FV, Freire C, et al. Brazilian IBD study group position statement on SARS-COV2 vaccination. Arq Gastroenterol 2021;58:1-4.

45. deBruyn J, Fonseca K, Ghosh S, et al. Immunogenicity of influenza vaccine for patients with inflammatory bowel disease on maintenance infliximab therapy: a randomized trial. Inflamm Bowel Dis 2016;22:638-647.

46. Melmed GY, Agarwal N, Frenck RW, et al. Immunosuppression impairs response to pneumococcal polysaccharide vaccination in patients with inflammatory bowel disease. Am J Gastroenterol 2010;105:148-154.

47. Pratt PK Jr, David N, Weber HC, et al. Antibody response to hepatitis B virus vaccine is impaired in patients with inflammatory bowel disease on infliximab therapy. Inflamm Bowel Dis 2018;24:380-386.

48. Andrade P, Santos-Antunes J, Rodrigues S, Lopes S, Macedo G. Treatment with infliximab or azathioprine negatively impact the efficacy of hepatitis B vaccine in inflammatory bowel disease patients. J Gastroenterol Hepatol 2015;30:1591-1595.

49. Park SH, Yang SK, Park SK, et al. Efficacy of hepatitis A vaccination and factors impacting on seroconversion in patients with inflammatory bowel disease. Inflamm Bowel Dis 2014; 20:69-74.

50. Agarwal N, Ollington K, Kaneshiro M, Frenck R, Melmed GY. Are immunosuppressive medications associated with decreased responses to routine immunizations? A systematic review. Vaccine 2012;30:1413-1424.

51. Wyant T, Leach T, Sankoh S, et al. Vedolizumab affects antibody responses to immunisation selectively in the gastrointestinal tract: randomised controlled trial results. Gut 2015;64: 77-83.

52. Andrisani G, Frasca D, Romero M, et al. Immune response to influenza $\mathrm{A} / \mathrm{H} 1 \mathrm{Nl}$ vaccine in inflammatory bowel disease patients treated with anti TNF- $\alpha$ agents: effects of combined therapy with immunosuppressants. J Crohns Colitis 2013;7: 301-307.

53. Sempere L, Almenta I, Barrenengoa J, et al. Factors predicting response to hepatitis B vaccination in patients with inflammatory bowel disease. Vaccine 2013;31:3065-3071.

54. Kim SB, Park SJ, Chung SH, et al. Vaccination and complementary and alternative medicine in patients with inflamma- tory bowel disease. Intest Res 2014;12:124-130.

55. Tinsley A, Navabi S, Williams ED, et al. Increased risk of influenza and influenza-related complications among 140,480 patients with inflammatory bowel disease. Inflamm Bowel Dis 2019;25:369-376.

56. Kantsø B, Simonsen J, Hoffmann S, Valentiner-Branth P, Petersen AM, Jess T. Inflammatory bowel disease patients are at increased risk of invasive pneumococcal disease: a nationwide danish cohort study 1977-2013. Am J Gastroenterol 2015;110: 1582-1587.

57. Farraye FA, Melmed GY, Lichtenstein GR, Kane SV. ACG clinical guideline: preventive care in inflammatory bowel disease. Am J Gastroenterol 2017;112:241-258.

58. Grohskopf LA, Alyanak E, Broder KR, et al. Prevention and control of seasonal influenza with vaccines: recommendations of the Advisory Committee on Immunization Practices. United States, 2020-21 influenza season. MMWR Recomm Rep 2020;69:1-24.

59. Kim KO, Jang BI. Management of inflammatory bowel disease in the COVID-19 era. Intest Res 2022;20:3-10.

60. Magro F, Rahier JF, Abreu C, et al. Inflammatory bowel disease management during the COVID-19 outbreak: the ten do's and don'ts from the ECCO-COVID Taskforce. J Crohns Colitis 2020; 14(14 Suppl 3):S798-S806.

61. Zambrano LD, Ellington S, Strid P, et al. Update: characteristics of symptomatic women of reproductive age with laboratory-confirmed SARS-CoV-2 infection by pregnancy status. United States, January 22-October 3, 2020. MMWR Morb Mortal Wkly Rep 2020;69:1641-1647.

62. Allotey J, Stallings E, Bonet M, et al. Clinical manifestations, risk factors, and maternal and perinatal outcomes of coronavirus disease 2019 in pregnancy: living systematic review and meta-analysis. BMJ 2020;370:m3320.

63. Kotlyar AM, Grechukhina O, Chen A, et al. Vertical transmission of coronavirus disease 2019: a systematic review and meta-analysis. Am J Obstet Gynecol 2021;224:35-53.

64. Rasmussen SA, Jamieson DJ. Pregnancy, postpartum care, and COVID-19 vaccination in 2021. JAMA 2021;325:1099-1100.

65. Steinhoff MC, Katz J, Englund JA, et al. Year-round influenza immunisation during pregnancy in Nepal: a phase 4, randomised, placebo-controlled trial. Lancet Infect Dis 2017;17:981-989.

66. Winter K, Nickell S, Powell M, Harriman K. Effectiveness of prenatal versus postpartum tetanus, diphtheria, and acellular pertussis vaccination in preventing infant pertussis. Clin Infect Dis 2017;64:3-8.

67. Adhikari EH, Spong CY. COVID-19 vaccination in pregnant 
and lactating women. JAMA 2021;325:1039-1040.

68. Society of Obstetricians and Gynaecologists of Canada. SOGC COVID-19 vaccination in pregnancy FAQ for health care providers [Internet]. c2021 [cited 2021 Jul 10]. https://sogc.org/ common/Uploaded\%20files/Covid\%20Information/EN_ HCP-FAQ_SOGC_FINAL.pdf

69. Centers for Disease Control and Prevention. Information about COVID-19 vaccines for people who are pregnant or breastfeeding [Internet]. c2021 [cited 2021 Jul 7]. https://www.cdc. gov/coronavirus/2019-ncov/vaccines/recommendations/ pregnancy.html.

70. U.S. Food and Drug Administration. Vaccines and Related Biological Products Advisory Committee meeting December 17, 2020: FDA briefing document Moderna COVID-19 vaccine [Internet]. c2021 [cited 2021 Jun 26]. https://www.fda. gov/media/144434/download.

71. Centers for Disease Control and Prevention. V-safe COVID-19 Vaccine Pregnancy Registry [Internet]. c2021 [cited 2021 Jul 7]. https://www.cdc.gov/coronavirus/2019-ncov/vaccines/ safety/vsafepregnancyregistry.html.

72. Shimabukuro TT, Kim SY, Myers TR, et al. Preliminary findings of mRNA COVID-19 vaccine safety in pregnant persons. N Engl J Med 2021;384:2273-2282.

73. Gray KJ, Bordt EA, Atyeo C, et al. Coronavirus disease 2019 vaccine response in pregnant and lactating women: a cohort study. Am J Obstet Gynecol 2021;225:303.e1-303.e17.

74. World Health Organization. Update on WHO Interim recommendations on COVID-19 vaccination of pregnant and lactating women [Internet]. c2021 [cited Jun 26]. https://www. who.int/publications/m/item/update-on-who-interim-recommendations-on-covid-19-vaccination-of-pregnant-andlactating-women.

75. Department of Health \& Social Care. Joint Committee on Vaccination and Immunisation: advice on priority groups for COVID-19 vaccination, 30 December 2020 [Internet]. c2020 [cited
2021 Jun 24]. https://www.gov.uk/government/publications/ priority-groups-for-coronavirus-covid-19-vaccination-advicefrom-the-jcvi-30-december-2020/joint-committee-on-vaccination-and-immunisation-advice-on-priority-groups-for-covid-19-vaccination-30-december-2020.

76. Public Health England. JCVI issues new advice on COVID-19 vaccination for pregnant women [Internet]. c2021 [cited 2021 Jul 5]. https://www.gov.uk/government/news/jcvi-issuesnew-advice-on-covid-19-vaccination-for-pregnant-women.

77. Martins I, Louwen F, Ayres-de-Campos D, Mahmood T. EBCOG position statement on COVID-19 vaccination for pregnant and breastfeeding women. Eur J Obstet Gynecol Reprod Biol 2021;262:256-258.

78. Medicines and Healthcare Products Regulatory Agency. UK regulator confirms that people should continue to receive the COVID-19 vaccine AstraZeneca [Internet]. c2021 [cited 2021 Jun 26]. https://www.gov.uk/government/news/uk-regulatorconfirms-that-people-should-continue-to-receive-the-covid-19-vaccine-astrazeneca.

79. Choi WS. Comprehensive understanding and field application of COVID-19 vaccine. Korean J Med 2021;96:155-159.

80. American College of Obstetricians and Gynecologists. Coronavirus (COVID-19), pregnancy, and breastfeeding: a message for patients [Internet]. c2021 [cited 2021 Jul 5]. https:// www.acog.org/womens-health/faqs/coronavirus-covid-19pregnancy-and-breastfeeding

81. Perl SH, Uzan-Yulzari A, Klainer H, et al. SARS-CoV-2-specific antibodies in breast milk after COVID-19 vaccination of breastfeeding women. JAMA 2021;325:2013-2014.

82. Royal College of Obstetricians and Gynaecologists. COVID-19 vaccines, pregnancy and breastfeeding [Internet]. c2021 [cited 2021 Jun 19]. https://www.rcog.org.uk/en/guidelines-research-services/coronavirus-covid-19-pregnancy-and-womens-health/covid-19-vaccines-and-pregnancy/covid-19-vaccines-pregnancy-and-breastfeeding/. 